\title{
Design and Research of Walking Judgment System of Horse Race along the Way
}

\author{
Shuang Zhang a, Ding Peng b, * \\ The International School of Equestrian, Wuhan Business University, Wuhan 430056, China. \\ azhangshuangyq@163.com, , , btiredhiker@hotmail.com
}

Keywords: horse race, real-time walk.

\begin{abstract}
The status of real-time walking judgment system in horse races at home and abroad is studied through the methods of literature consultation, expert interview method and field investigation method in this paper. The result shows that the judgment system equipment of walking along the way currently used in the market are manual operations, there are complicated operation processes in the manually operated equipment, require a large amount of manpower, and have the disadvantages such as delay and accuracy. For this reason, this research has specially designed an automated real-time judgment system for horse race along the way; this system can judge the real-time position information of horses in the race quickly and accurately, the application of this system can provide effective reference data for horse race guess, provide data reference for horse trainers to teach horses, provide information experience for walking decision-making in jockey races, and provide technical support for the development of the horse racing industry.
\end{abstract}

\section{Introduction}

Horse race real-time walking judgment system is one kind of equipment for measuring the position context of horses in races. This equipment has the characteristics of professionalism and scarcity, according to the survey, there are few equipment sold on the market. Because of the particularity of this equipment, if the race courses need this equipment at home and abroad, the horse race courses can cooperate with professional companies to develop and customize according to the needs of its own enterprise, the equipment produced by this cooperation mode is usually very expensive. At present, the real time walking judgment systems used by most horse courses are manual operation system, the achievement of this manual operation system needs to be completed by software, and does not involve too much external hardware, the required cost only the cost of software development, so the price is more reasonable and can be accepted for most race courses. However, this manual operation software is tedious in operation process and low accuracy and is influenced by the operator's reaction speed and action speed. In the contemporary era of information popularization, it is necessary to solve the problem of solve the problem of horse walking on the horse race from the point of view of information to adapt to the rapid development of the China's race horse industry, for this reason, this research specially designs an automated real-time walking judgment system, simplifies the operation process, improves the measurement precision and speed, for the development of the race horse industry. Provide high quality technical service support.

\section{Introduction of Manual Real-time Walking Judgment System}

\subsection{Basic Principle of Manual Real-Time Walking Judgment System.}

The manual real-time walking judgment system is implemented by software, this software needs to be operated on the ordinary computer, and the real-time walking information of horse is displayed on the LED screen of the racecourse through computer operation. The operation of this system requires two staffs to cooperate with each other. One operator is responsible for the operation of the computer and the other operator (also called as an observer) needs to observe the order of the horses in the race through a telescope, because of the limited memory and judgment of the person, the order of the first 
6 order is generally judged. The computer operator can only concentrate on the operation of the walking judgment software; there is no time to observe the horse conditions o in the racecourse, the observer observes the real-time position of the horses in the race through the telescope, and does not have time to carry out computer operations, in order to complete real-time walking information in the racecourse, the two parties need to communicate in a timely and accurate way through language, any error in either of the two will cause the recording of the position information of the horse in the race. After the start of the race, it is difficult for the horses to pull apart from each other in a short time. After the start of the race, it is very difficult for horses to apart from each other in a short time, it is usually 100 meters to 200 meters after the opening of the gate, and the distance among horses will increase, at this time, the observer observes the positional relationship among the horses through the telescope, inform the computer operator of the position information, the computer operator quickly presses the horse's number and sends the position information to the LED screen for display. The main problem of the manual real-time walking judgment system is that the positional relationship of the horses in the race changes frequently, which requires the observers to be highly concentrated and timely update and report the position information to the computer operator. Because the horse racing time is very short, the horses run faster, therefore, it is difficult to quickly and accurately judge the position information of the horses and display on the LED screen.

\subsection{Defects in Manual Real-Time Walking Judgment System.}

There are the following several disadvantages in the manual real-time walking judgment system. First: more operators are needed; the entire race needs two staff members to work together to complete it, which will cause labor and expense costs for the entire event to increase. Second: lack of precision and speed; the operation manual real-time walking judgment system belongs to manual operation, the accuracy of the time is affected by the computer operator's reaction speed and action speed, but also affected by the observer's reaction speed, there are more horses in the same game, and it is difficult for observers to quickly judge the positional information among horses. Third: the error is relatively large; because the observer is standing in a fixed position to observe the real-time position of the horses on the racecourse, and the track is oval, there is a large visual error in observing the fast moving horses in the race through the fixed points, causes the observed result to be inconsistent with the actual result. Fourth: due to the slow response of people, the speed which horses run is faster, and there are more horses in the race and the rankings in the race change frequently, therefore, it is difficult to accurately judge the real-time position information of the horses in the race.

\section{Automatic Real-time Walking Judgment System Design}

\subsection{Design Principles of Automatic Real-Time Walking Judgment System.}

The automatic real-time walking judgment system adopts informational means to solve the positional relationship problems among horses in the race. This system needs to place an electronic chip inside each horse's nasal hoops, the role of this chip is to send the real-time position information of the horses, and the sent distance should not be less than $1 \mathrm{~km}$. The received equipment for real-time position information is installed in the terminal control room for receiving position information of each horse in the race. The updated frequency of chip emitter is hundredths of a second, and the information receive equipment can do real-time sorting according to the position information of each horse and can keep pace with the LED display screen at any time. The completion of the entire process requires only opening and closing the software.

\section{2 function Modules of Automatic Real-Time Judgment System.}

\subsubsection{Position Chip Emitter.}

The position chip emitter is installed inside the horse's nasal hoop and is used to send the horse's real-time position information. Because the ranking judgment of horses is based on the forefront part of the horse's nose, therefore, the position chip emitter is installed on the horse's nasal hoop can reduce errors and improve accuracy. Because the position chip emitted device is an electronic device and needs to have a charging function, therefore, the chip can be set to be pluggable. When the 
position chip emitter device power is insufficient, the emitter can be charged or replaced before the race begins. In addition, the position chip emitter has the function of acquiring its own position through the GPS positioning function, and the position error is less than $10 \mathrm{~cm}$.

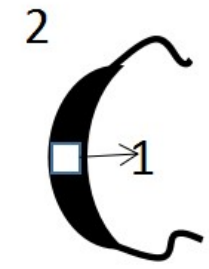

1. Pluggable positioning chip; 2. Nasal hoop

Fig. 1 Nasal hoop and positioning chip

\subsubsection{Information Receiving Device.}

The information receiving device is used to receive the position signal transmitted from the position chip emitted at any time and transmit the signal to the information service station. The range which information receiving device is capable of receiving signals is not less than $1 \mathrm{~km}$ and has a high sensitivity. The information receiving device is transmitted to the information service station via the USB connection. The information receiving device pattern is shown in Figure 2.

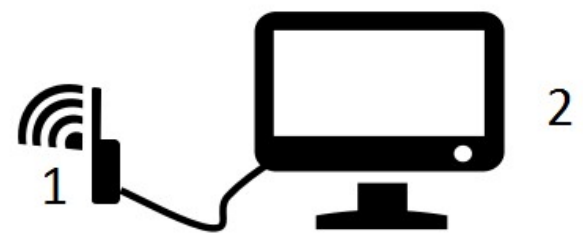

1. Information receiving device; 2 . Information service station

Fig. 2 Information receiving device

\subsubsection{Information Service Station.}

The information service station is used to process, store, query, and display the position information of all the horses. The information service station includes the following function modules: horse information input module, horse position information processing and display module, and position information query and processing module of historical results. The horse information input module includes horse name, saddle number, branding number, owner, stud-farm, competition time, competition position, competition schedule and other information, this information is mainly used for query the past results of the horse and build index. The horse position information processing and display module is used to process the position data information transmitted by the position chip; this module can quickly sort the order of the horses on the field according to the position data and can transmit the ranking information to the LED display screen at any time. The position information query and processing module of historical results is used to query the ranking information of each horse in previous races, it can be quickly queried based on the time, frequency, horse name, saddle number, branding number, owner, stud-farm, and other indexes. The pattern diagram of the information service station is shown in Figure 3.

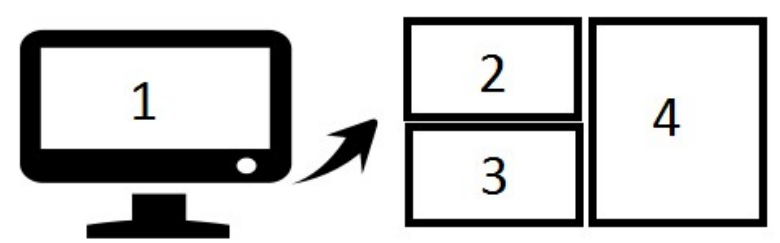

1. Information service station; 2. Horse position information processing and display module; 3. Horse information input module; 4 . Position information query and processing module in historical results

Fig. 3 Information service station 


\subsubsection{Pattern Diagram of Working Principles.}

The schematic diagram of the principle of automatic real-time walking judgment system coordination work is shown in Figure 4.

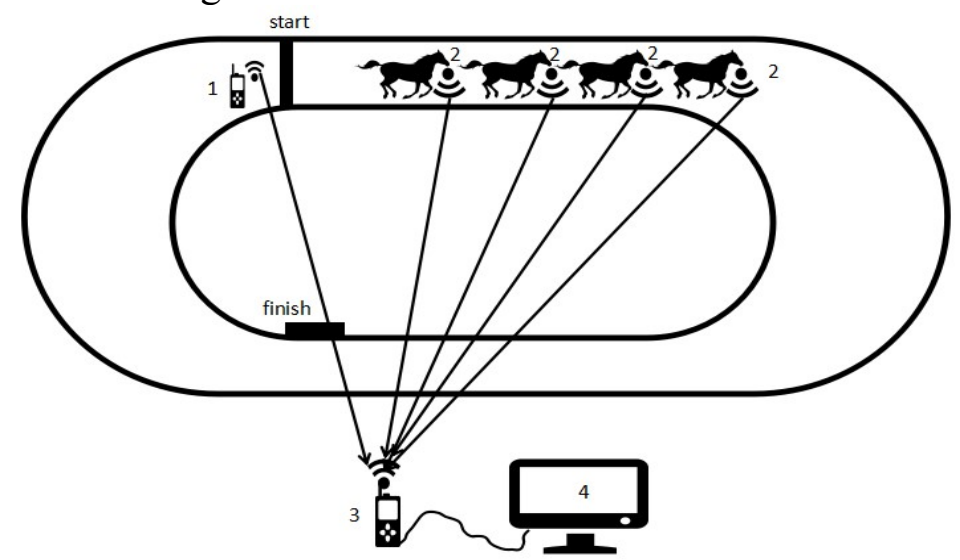

1. Starting signal emitter; 2. Position launcher; 3. Information receiving device; 4. Information service station;

Fig. 4 coordination working mode of automatic real-time walking judgment system

\section{The Advantages and Application of Automatic Real-Time Walking Judgment System}

\subsection{Advantages of Automatic Real-Time Walking Judgment System}

The advantages of the automatic real-time walking judgment system is that a positioning chip is set on each horse's nasal hoops, and the position data of the horses in the race can be sent to the information service station in real time, thereby realizing the automatic processing of rankings in the horse races, reducing the workload and improving accuracy and speed. At the same time, the automatic real-time walking judgment system has a data storage function, which can store the position information of all participating horse races in a database, and can acquire data information at any time according to needs, and provide data support for horse racing ability analysis, scientific research, and guess activities, etc.

\subsection{Application of Automatic Real-Time Walking Judgment System}

The position information recorded by the real-time walking judgment system in the horse race is mainly used in the analysis of the horse's athletic ability, horse racing guess, the daily training of the horses, and the walking decision-making reference in the jockey competition. First: Analysis of horses' athletic ability. Through the walking data information in the horse races, the horse's movement characteristics can be understood and the running types of the horses can be distinguished, secondly, horse racing guess. Horse fans can understand the horse's race characteristics through the information of the horse's walking data in horse race, at the same time, the power condition of the horse can be judged based on the horse's past ranking information, according to these time information, choose the right horse to bet; third: The daily training of horses. According to the walking date information in the horse races, the training amount of horses can be adjusted, and improve the running method of the competition; fourth, the reference of the walking decision-making in jockey competitions. The jockey can determine how to ride a horse in the race according to the walking data information in the horse race, how to accelerate and decelerate, and provide data references for the jockey's walking decision-making in the race.

\section{Conclusion and Suggestion}

There are more operators in manual position judgment equipment, lack accuracy and speed, and it is not possible to measure the ranking information of all the participating horses in the race. The manual walking judgment equipment is no longer suitable for use in racing events. 
Automatic real-time walking position judgment system has automatic processing, reduced workload, high accuracy, high speed, etc. It is the future development trend.

The real-time walking judgment system has a very important role for horse race guess, horse trainers, jockeys and other personnel. It can provide horse fans with reference data, provide trainers with training intensity indexes, and provide jockeys with help in walking strategies in horse races.

It is suggested that all events in the country are organically linked, a national database platform is established, can carry out save and statistical analysis for the walking data information of all the horses in the events along the way at any time, and provide technical services for the national horse races.

\section{References}

[1]. Wang Lingo. Research on multi-section high-speed CCD timing system for track training [J]. Journal of Sports Computer Application, 2010.3:813-814.

[2]. Xing Yu. Theory and practice of high-level sprint training based on precise segmented timing [J]. Journal of Chengdu Sport University, 2014. 6:51-56.

[3]. Jib Gushing. Design and achievement of manual segmented timing method for straight sprint [J]. Athletics.2015.12:47-49.

[4]. Ma Magda. Preliminary study on sports lottery in Macau [J]. Sports Culture Guide [J]. 2006.4

[5]. Wang Hong, Hong Haikou, Qi Chinghai. Review and development trend of sports lottery industry [J]. Journal of Guangzhou Sport University.2000.3

[6]. Wu Yang, The enlightenment development of sports lottery in the United States on the development of sports lottery in China [J]. Sports Culture Guide.2003.11.

[7]. $\mathrm{Cu} \mathrm{Na}$, Li Hay, Zing Howie, etc. Research on social responsibility of Internet sports lottery Company [J]. Journal of Shenyang Sport University. 2011.4. 